\title{
INFECÇÃO DE Conotrachelus humeropictus FIEDLER (COLEOPTERA: CURCULIONIDAE) POR Metarhizium anisopliae (METSCH.) SOR. E Beauveria bassiana (BALS.) VUILL. NO SOLO
}

\author{
Antonio C. B. MENDES' 1 , Bonifácio P. MAGALHÃES ${ }^{2}$, Orlando S. OHASHI ${ }^{3}$, \\ Cleber N. BASTOS ${ }^{1}$
}

RESUMO - Foi avaliada a infecção de larvas de Conotrachelus humeropictus Fiedler, séria praga do cacaueiro (Theobroma cacao L.) e do cupuaçuzeiro (T. grandiflorum (Willd. ex Spreng.) Schum.) na Amazônia brasileira, por Metarhizium anisopliae (Metsch.) Sor, e Beauveria bassiana (Bals.) Vuill, A pesquisa foi desenvolvida nos campos experimentais da Comissão Executiva do Plano da Lavoura Cacaueira - CEPLAC, em Ouro Preto D'Oeste, Rondônia. Foram testadas suspensões de $3,93 \times 10^{10}$ conídios $/ \mathrm{ml}$ de $M$. anisopliae e $4,26 \times 10^{10}$ conídios $/ \mathrm{ml}$ de $B$. bassiana, pulverizadas superficialmente em solo contido em recipientes de PVC, onde em diferentes dias após a pulverização (um, três, sete e quatorze dias) liberou-se larvas do último instar da praga. Beauveria bassiana mostrou-se mais eficiente $(52,0 \%$ de mortalidade) do que $M$. anisopliae $(42,7 \%)$, evidenciando assim, seu maior potencial no controle da praga. Os indices de mortalidade foram estatisticamente iguais para larvas liberadas até o $7^{\circ}$ dia da contaminação, decrescendo significativamente no $14^{\circ}$ dia. A queda na efetividade pode estar associada à presença de microrganismos antagonistas no solo.

Palavras-chave: Controle biologico, insecta, Theobroma cacao, Theobroma grandiflorum

Infection of Conotrachelus humeropictus Fiedler (Coleoptera: Curculionidae) by Metarhizium anisopliae (Metsch.) Sor. and Beauveria bassiana (Bals.) Vuill. in the Soil

\begin{abstract}
The infection of Conotrachelus humeropictus Fiedler, a serious pest of cocoa and cupuaçu fruit in the Brazilian Amazon, by Metarhizium anisopliae and Beauveria bassiana was evaluated. The research was conducted in a field station of the Comissão Executiva do Plano da Lavoura Cacaueira - CEPLAC, in Ouro Preto d'Oeste, Rondonia. Suspensions of $3.93 \times 10^{10}$ conidia $/ \mathrm{ml}$ of $M$. anisopliae and $4.26 \times 10^{10} \mathrm{conidia} / \mathrm{ml}$ of $B$. bassiana were superficially sprayed on soil in PVC flasks. One, three, seven and fourteen days after spraying the last larval instar were released. Beauveria bassiana was more eficient ( $52.0 \%$ mortality) than $M$. anisopliae $(42.7 \%)$. The mortalities were the same for larvae liberated until the $7^{\text {th }}$ day after contamination, and were significantly lower on the $14^{\text {th }}$ day. The decline in the efficacy of the pathogens may be related to the presence of antagonic organisms in the soil.
\end{abstract}

Key-words: Biological control, insecta, Theobroma cacao, Theobroma grandiflorum

\section{INTRODUÇÃO}

Conotrachelus humeropictus Fiedler é considerada uma das principais pragas do cacaueiro, Theobroma cacao L., na Amazônia brasileira, sendo um dos fatores responsáveis pela desclassificação e depreciação da qualidade intrínseca e extrínseca das amêndoas na região (Mendes et al., 1988). Além dessa cultura, ataca os frutos de cupuaçu, Theobroma grandiflorum (Willd. ex Spreng.) Schum. (Trevisan \& Mendes,

\footnotetext{
'CEPLAC/Superintendência Regional da Amazônia Oriental, C. Postal 1801, 66.635-110, Belém, PA, email:acbm@ufpa.br

2EMBRAPA/CENARGEN, C. Postal, 02372, 70.849-970, Brasilia, DF, boni@embrapa.cenargen.gov.br ${ }^{3}$ FCAP/Departamento de Biologia Vegetal e Fitossanidade, C. Postal 917, 66.077-530, Belém, PA
} 
1991; Laker \& Trevisan, 1992; Venturieri, 1993), tornando sua polpa, muito apreciada e utilizada para sucos e doces, imprestável para o consumo.

Aspectos do comportamento desta praga no campo foram apresentados por Trevisan (1969), tendo Mendes et al. (1997) elucidado parâmetros da biologia. Constataram que o inseto, abandonando o fruto, permanece no solo por um periodo médio de 20,25 dias, para completar as fases de pré-pupa, pupa e maturação fisiológica do adulto recém-formado, estando nesse periodo, mais vulnerável a ação de seus inimigos naturais.

O controle de C. humeropictus hoje utilizado se restringe exclusivamente ao uso de produtos químicos, com resultados duvidosos e, principalmente, antieconômicos, em decorrência do número elevado de aplicações do inseticida. Além desses aspectos, deve-se considerar os problemas de poluição ambiental que resultam em sérias conseqüências com relação aos prováveis residuos em amêndoas e destruição da fauna benéfica. Por estes motivos, a busca de outros métodos de controle deve ser intensificada, de modo a reduzir a população do inseto a niveis de danos não econômicos.

Visando o controle de curculionídeos que passam pelo menos uma fase do seu ciclo no solo, os fungos entomopatógenos Metarhizium anisopliae e Beauveria bassiana foram intensivamente testados, como para o controle de Chalcodermus aeneus (Bell \& Hamalle, 1970; Quintella, 1986), Curculio caryae (Horm) (Tedders et al., 1973; Gottwald \& Tedders, 1983, 1984), Conotrachelus nenuphar (Tedders et al., 1982) e de Conotrachelus bimaculatus Boh. (Quintella \& Roberts, 1992; Quintella et al., 1990, 1992, 1994).

A constatação de larvas de $C$. humeropictus infectadas pelos entomopatógenos $M$. anisopliae e $B$. bassiana na região de Ouro Preto D'Oeste, RO, e a potencialidade apresentada por esses fungos em laboratório para o controle da praga (Bastos et al., 1988; Mendes, 1996), aliados ao hábito do inseto e ao ambiente favorável aos entomopatógenos no solo do agroecossistema cacaueiro, abriram perspectivas para o seu controle microbiano. Esse método, por sua compatibilidade, especificidade e segurança ao homem e a outras formas de vida, destaca-se como um dos mais promissores, principalmente quando associado a outras táticas de manejo integrado de pragas.

Assim, o presente trabalho teve por objetivos avaliar a infecção de larvas de $C$. humeropictus em diferentes períodos após a aplicação superficial dos entomopatógenos $M$. anisopliae e B. bassiana no solo.

\section{MATERIAL E MÉTODOS}

Sob uma lavoura cacaueira da Estação Experimental da Comissão Executiva do Plano da Lavoura Cacaueira 
em Ouro Preto D‘Oeste, RO (CEPLAC/ ESEOP), foram distribuidos na superficie do solo recipientes de PVC $(15 \mathrm{~cm}$ de diâmetro x $20 \mathrm{~cm}$ de altura) com fundo de tela de $1 \mathrm{~mm}$ de abertura e tecido de náilon. Estes recipientes continham em mais da metade de sua capacidade mistura de solo superficial e terra roxa estruturada eutrófica, retirada da própria lavoura e com a composição microbiológica prédeterminada. Sobre esta mistura de solo foram aplicados $7,0 \mathrm{ml}$ de suspensões em água com

Tween $0,05 \%$ de $M$. anisopliae e $B$. bassiana, correspondendo aproximadamente a 150 litros por hectare, nas concentrações de $3,93 \times 10^{10} \mathrm{e}$ $4,26 \times 10^{10}$ conídios viáveis $/ \mathrm{ml}$, respectivamente, determinadas como as de melhores performances para o controle da praga por Mendes (1996).

A viabilidade dos conídios foi obtida adicionando-se 10 microlitros de uma suspensão com aproximadamente $10^{4}$ conídios $/ \mathrm{ml}$, em placas de Petri com meio BDA. A suspensão foi espalhada por toda a superfície das placas, que, em seguida foram colocadas em estufa incubadora a $26^{\circ} \mathrm{C}$ de temperatura e fotofase de 12 horas. A avaliação dos conidios germinados e não germinados foi feita 16 horas após a incubação, observando-se 100 conídios de cada placa sob microscópio.

Após a aplicação dos entomopatógenos, com pulverizador costal, foram introduzidas em cada recipiente cinco larvas de $C$. humeropictus no último ínstar, totalizando dez larvas por parcela, da seguinte maneira: a) larvas distribuidas um dia após a aplicação dos fungos; b) larvas distribuídas no $3^{\circ}$ dia após; c) larvas distribuídas no 7ำ dia após e d) no $14^{\circ}$ dia após. Os recipientes testemunha receberam pulverizações de água com Tween $0,05 \%$ na mesma quantidade que os tratamentos entomopatogênicos. O delineamento estatístico seguiu o esquema fatorial $3 \times 4 \mathrm{em}$ blocos, onde os fatores estudados consistiram das espécies fúngicas e dos diferentes períodos de introdução das larvas, com cinco repetições. Transcorrido o período de dez dias, para cada período de introdução, foram registrados os insetos vivos e reconhecidamente mortos pelos fungos no interior dos recipientes, os quais encontravam-se cobertos, desde a distribuição das larvas, com tecido de náilon, preso com ligas de borracha. Ao final dos ensaios, realizou-se a análise microbiológica qualitativa do solo contido nos recipientes, segundo o método de Nader (1992), utilizando-se meio extrato de malte-agar (EMA).

\section{RESULTADOS E DISCUSSÃO}

Os dois entomopatógenos revelaram índices de mortalidade significativamente diferentes entre os grupos tratados e a testemunha. Os valores médios de mortalidade de $C$. humeropictus devida a $B$. bassiana e $M$. anisopliae foram de $52,0 \%$ e $42,7 \%$, respectivamente.

Os entomopatógenos provocaram indices de mortalidade similares para larvas liberadas até o $7^{\circ}$ dia da pulverização do solo contido nas parcelas. Entretanto, no solo em que as larvas foram liberadas quatorze dias após a pulverização, os valores 
decresceram de forma significativa, sugerindo assim uma tendência linear, tanto para $M$. anisopliae $(\mathrm{F}=23,90$; $\left.\mathrm{R}^{2}=0,86 ; \mathrm{a}=0,01\right)$ como para $B$. bassiana $\left(\mathrm{F}=50,91 ; \mathrm{R}^{2}=0,99\right.$; $\mathrm{a}=0,01)$ (Tab. 1; Fig. 1).
Os índices de mortalidade obtidos estão abaixo daqueles determinados por Tedders et al. (1982) para C. nenuphar, onde, diferentemente da presente pesquisa, M. anisopliae provocou um porcentual

Tabela 1. Mortalidade média (\%) de Conotrachelus humeropictus, após liberação das larvas em diferentes dias, em solo pulverizado com suspensões de conídios de Metarhizium anisopliae e Beauveria bassiana em Ouro Preto D'Oeste, RO.

\begin{tabular}{lccccc}
\hline Tratamento & Concentração & \multicolumn{4}{c}{ Dias de liberação das larvas após pulverização } \\
\cline { 3 - 6 }$($ conídios $/ \mathrm{ml})$ & $4,26 \times 10^{10}$ & $66,37 \mathrm{aA}$ & $60,17 \mathrm{aA}$ & $52,01 \mathrm{aA}$ & $29,80 \mathrm{aB}$ \\
\hline B. bassiana & $4,93 \times 10^{10}$ & $52,10 \mathrm{aA}$ & $45,89 \mathrm{aA}$ & $47,99 \mathrm{aA}$ & $25,84 \mathrm{aB}$ \\
M anisopliae & $3,9,95 \mathrm{bA}$ & $6,48 \mathrm{bA}$ & $7,95 \mathrm{bA}$ & $10,00 \mathrm{bA}$ \\
Testemunha & & $7,45 \%=11,42$
\end{tabular}

${ }^{1}$ Médias seguidas por letras distintas em colunas (minúsculas) e em linhas (maiúsculas) diferem entre si ao nivel de significância de $5 \%$ pelo teste de Tukey. Para a análise estatística, as médias foram transformadas em arc sen

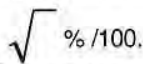

${ }^{2}$ Mortalidade natural.

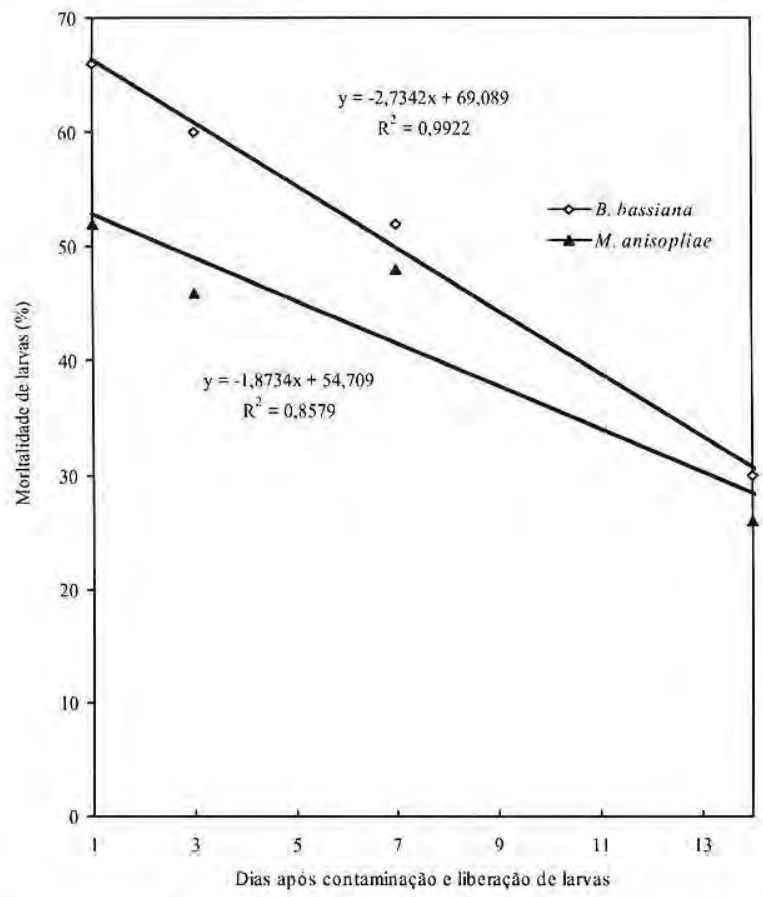

Figura 1. Porcentagem média de mortalidade de Conotrachelus humeropictus, liberados em diferentes dias após contaminação do solo com Metarhizium anisopliae e Beauveria bassiana. 
de mortalidade que variou de 82 a $100 \%$, mostrando-se mais efetivo, inclusive, do que $B$. bassiana. Para $M$. anisopliae, não houve discordância quanto ao índice de mortalidade de larvas liberadas em diferentes dias, quando os autores verificaram não haver diferença significativa quanto à liberação após um, três, cinco e sete dias da contaminação do solo. Contudo, para B. bassiana os índices são conflitantes, já que os autores determinaram que as larvas liberadas um dia e sete dias após a contaminação apresentaram, respectivamente, a menor $(26 \%)$ e a maior (82\%) mortalidade.

Resultados semelhantes quanto a esse aspecto também foram apresentados por Quintela et al. (1994) para C. bimaculatus, onde a eficiência dos entomopatógenos não diminuiu seis dias após aplicação, sendo o número de insetos sobreviventes, dentro de cada tratamento, significativamente similar nas diferentes datas de liberação das larvas (imediatamente após a aplicação no solo, três, cinco e seis dias após). A eficiência média de controle encontrada pelos autores, em Goiás, foi de $50 \%$ para $M$. anisopliae e $B$. bassiana, próximo aos resultados da presente pesquisa.

O decréscimo da mortalidade de $C$. humeropictus, nas parcelas em que as larvas foram liberadas quatorze dias após a pulverização, pode ter sido favorecido pela presença de prováveis antagonistas constatados no solo no início do experimento (Tab. 2), entre os quais os fungos Penicillium citrinum Thom, Penicillium citreonigrum Thom, Aspergillus versicolor (Vuill.) Tiraboschi e Gliocladium virens Miller, além de bactérias não identificadas. Estes organismos foram constatados por Mendes (1996) como principais antagônicos, in vitro, desses entomopatógenos.

O declínio acentuado de $M$. anisopliae e $B$. bassiana, em solos de cerrado de Goiás e do sertão semi-árido do Ceará, após seis e nove dias da aplicação dos entomopatógenos, também foi constatado por Quintela et al. (1992, 1994). Em avaliações seguintes, verificaram a estabilidade desses entomopatógenos, especialmente B. bassiana. Quintela et al. (1994) atribuiram ao rápido declínio e à baixa recuperação de $M$. anisopliae, a presença de Aspergillus no solo. Majchrowicz et al. (1990) se referiram a espécie de Aspergillus clavatus Link como antagônica à $B$. bassiana, e Lingg \& Donaldson (1981) isolaram e comprovaram o efeito fungistático de Penicillium urticae sobre esse entomopatógeno.

Antagônicos presentes no solo têm sido referidos, entre outros, como responsáveis pela baixa persistência desses entomopatógenos neste substrato (Lingg \& Donaldson, 1981; Oliveira et al., 1981; Fargues et al., 1983; Gottwald \& Tedders, 1984; McCoy et al., 1992; Pereira et al., 1993). Entretanto, os resultados aqui obtidos revelaram persistência, já que 24 dias após a única aplicação $M$. anisopliae e $B$. bassiana ainda estavam presentes no solo (Tab. 2) e foram capazes de matar 25,8 e $29,8 \%$ dos insetos-alvo liberados quatorze dias após a contaminação das parcelas pelos inóculos. 
Tabela 2. Microrganismos presentes no solo de Ouro Preto D'Oeste, RO, antes (inicial) e 24 dias (final) após pulverização com suspensões de conídios Metarhizium anisopliae e Beauveria bassiana.

\begin{tabular}{ll}
\hline \multicolumn{1}{c}{ Microrganismo (Inicial) } & \multicolumn{1}{c}{ Microrganismo (Final) } \\
\hline Aspergillus versicolor (Vuill.) Tiraboschi & Aspergillus sp., \\
Fusarium sp. & Beauveria bassiana (Bals.) Vuill. \\
Gliocladium candidum Link ex Pers & Gliocladium candidum \\
Gliocladium virens Miller & Gliocladium virens Miller \\
Lasiodiplodia sp. & Gliocladium viride \\
Penicillium citrinum Thom & M.etarhizium anisopliae (Metsch.) Sor. \\
Penicillium citreonigrum Thom & Penicillium citrinum Thom \\
Penicillium sp., & Penicillium citreonigrum Thom \\
Penicillium sp., & Penicillium sp. \\
Penicillium sp., & Penicillium sp. \\
Thielaviopsis paradoxa Went., & Penicillium sp. \\
Tricoderma harzianum Rifai & Tricoderma harzianum Rifai \\
Tricoderma polysporum (Link. ex Pers.) Rifai & Bactéria n. det. \\
Trichoderma sp. & \\
Streptomyces sp. (Actinomiceto) & \\
Bactéria n. det. & \\
\hline
\end{tabular}

Os resultados sugerem que os entomopatógenos $M$. anisopliae e $B$. bassiana apresentam perspectivas de controle da broca $C$. humeropictus, quando pulverizados no solo sob a forma de conídios, embora apresentando um decréscimo de eficácia, após sete dias da pulverização, atingindo os menores indices aos quatorze dias. $\mathrm{Na}$ concentração utilizada, Beauveria bassiana apresentou-se mais virulento que $M$. anisopliae, evidenciando seu maior potencial. Pesquisas utilizando o fungo em larga escala no campo devem ser incrementadas, inclusive em unidades de validação, antes da sua recomendação ńo controle da praga. Os dados evidenciam também que atenção deve ser dada à ação antagônica de microrganismos presentes no solo, quando da utilização desses entomopatógenos.

\section{AGRADECIMENTOS}

Aos Drs. José Luiz Bezerra, pesquisador da CEPLAC, pela determinação das espécies fúngicas, $\mathrm{e}$ ao Tec. Agícola Francisco Antonio Neto, da CEPLAC/ESEOP, pelo auxílio na coleta dos dados.

\section{Bibliografia citada}

Bastos, C.N.; Mendes, A.C. de B.; Garcia, J. de J. da S. 1988. Patogenicidade de Metarhyzium anisopliae (Metch.) e Sorokin e Beauveria bassiana (Bals.) Vuill. Sobre larvas de Conotrachelus sp. 
(Coleoptera: Curculionidae), broca dos frutos do cacaueiro (Theobroma cacao L.). Rev. Theobroma, 18(2):159-163.

Bell, V.J.; Hamalle, J.R. 1970. Three fungi tested for control of the cowpea curculio Chalcodermus aeneus. J. Invertebr. Pathol., 15:447-450.

Fargues, J.; Reisinger, O.; Robert H.P,; Aubart, C. 1983. Biodegradation of entomopathogenic hypomycetes: influence of clay coating on Beauveria bassiana blastospore survival in soil. J. Invertebr. Pathol., 41:131-142.

Gottwald, R.T.; Tedders, L.W. 1983. Supression of pecan weevil (Coleoptera: Curculionidae) populations with entomopathogenic fungi. Environ. Entomol., 12:471-474.

Gottwald, R.T; Tedders, L.W. 1984. Colonization transmission and longevity of Beauveria bassiana and Metarhizium anisopliae (Deuteromycotina: Hypomycetes) on pecan weevil larvae (Coleoptera: Curculionidade) in the soil. Environ. Entomol., 13:550-557.

Laker, A.H.; Trevisan, O. 1992. The increasing importance of cupuassu (Theobroma grandiflorum) (Schum.) in the Amazon Region of Brazil. Cocoa Growers' Bull., $45: 45-52$.

Lingg, J, ${ }^{\mathrm{a}}$; Donaldson, D.M. 1981. Biotic and abiotic factors affecting stability of Beauveria bassiana conidia in soil. $J$. Invertebr. Pathol., 39:191-200.

Majchrowicz, I.; Poprawski, J.T.; Maniania, K.N.; Robert, H.P. 1990. Effects of entomopathogenic and opportunistic fungi on Delia antiqua (Diptera: Anthomyiidae) at low relative humidity. Environ. Entomol., 19:1163-1167.

McCoy, C.W.; Storey, G.K.; Tigano-Milani, M.S. 1992. Environmental factors affecting entomopathogenic fungi in the soil. Pesq. Agrop. Bras., 27:107-111.

Mendes, A.C. de B. 1996. Biologia e controle microbiano de Conotrachelus humeropictus Fiedler, 1940 (Coleoptera: Curculionidae). Tese de Doutorado, Universidade Federal do Pará/Museu Paraense Emílio Goeldi, Belém, Pará. 101 p.

Mendes, A.C. de B.; Ribeiro, N.C.; Garcia, J. de J. da S.; Trevisan, O. 1988. Danos de Conotrachelus humeropictus Fiedler, 1940
(Coleoptera: Curculionidae): Nova praga do cacaueiro (Theobroma cacao L.) na Amazônia brasileira. An. Soc. Entomol. Brasil., 17:19-28.

Mendes, A.C. de B.; Magalhães, B.P.; Ohashi, O.S. 1997. Biologia de Conotrachelus humeropictus, Fiedler, 1940 (Coleoptera: Curculionidae), praga do cacaueiro e cupuaçuzeiro na Amazônia brasileira. Acta Amazonica, 27(2):135-144.

Nader, R.N. 1992. Microbiologia. Manual de laboratório. Nobel, São Paulo. 138p.

Oliveira, D.P.; Chaves, G.M.; Loures, E.G. 1981. Estudo comparativo da sobrevivência de Metarhizium anisopliae (Metsch.) Sorokin em diferentes tipos de solo. Rev. Theobroma, 11:233-239.

Pereira, R.M.; Stimac, J.L.; Alves, S.B. 1993. Soil antagonism affecting the dose-response of workers of the imported red fire ant, Solenopsis invicta, to Beauveria bassiana conidia. J. Invertebr. Pathol., 61:156-61.

Quintela, E.D. 1986. Estabilidade de Beauveria bassiana (Bals.) Vuillemin (Hyphomycetes) no solo e sua patogenicidade ao Chalcodermus aeneus Boheman (Coleoptera: Curculionidae), praga do caupi. Dissertação de Mestrado, Escola Superior de Agricultura Luiz de Queiroz/Universidade de São Paulo, Piracicaba. $107 \mathrm{p}$.

Quintela, E.D.; Lord, J.C.; Wraight, S.P.; Alves, S.B.; Roberts, D.W. 1990. Pathogenicity of Beauveria bassiana (Hyphomycetes: Moniliales) to larval adult Chalcodermus bimaculatus (Coleoptera: Curculionidae). J. Econ. Entomol., 83:1276-1279.

Quintela, E.D.; Roberts, D.W. 1992. Controle de Chalcodermus bimaculatus (Boheman) (Coleoptera: Curculionidae) no solo com Beauveria bassiana (Bals.) Vuillemin e Metarhizium anisopliae (Metsch.) Sorokin. Pesq. Agropec. Bras., 27:95-105.

Quintela, ED.; Lord, J.C.; Alves, S.B.; Roberts, D.W. 1992. Persistência de Beauveria bassiana em solo de Cerrado e sua interação com microorganismos do solo. An. Soc. Entomol. Brasil, 21:69-82.

Quintela, ED.; Wraight, S.P.; Quindaré, M.A.W.; Roberts, D.W. 1994. Aplicação de conidios de Beauveria bassiana (Bals.) Vuill. e Metarhizium anisopliae (Metsch.) 
Sor. para controle de larvas de Chalcodermus bimaculatus Boh. (Coleoptera: Curculionidae) no solo. $A n$. Soc. Entomol. Brasil., 23:367-77.

Tedders, W.L.; Weaver, D.J.; Wehunt, E.J. 1973. Pecan weevil supression of larvae with the fungi Metarhizium anisopliae and Beauveria bassiana and the nematode Neoaplectana dutki. J. Econ. Entomol., 66:723-25.

Tedders, W.L.; Weaver, D.J.; Wehunt, E.J.; Gentry, C.R. 1982. Bioassays of Metarhizium anisopliae, Beauveria bassiana, and Neoplectana carpocapsae against larvae of the plumcurculio Conotrachelus nenuphar (Herbst) (Coleoptera: Curculionidae). Environ. Entomol., 11:901-904.

Trevisan, O, 1989. Comportamento da broca dos frutos, Conotrachelus humeropictus Fiedler, 1940 (Coleoptera: Curculionidae), em Rondônia. Dissertação de Mestrado, Escola Superior de Agricultura Luiz de Queiroz/Universidade de Sào Paulo, Piracicaba. 75 p.

Trevisan, O.; Mendes, A.C. de B. 1991. Ocorrência de Conotrachelus humeropictus Fiedler, 1940 (Coleoptera: Curculionidae) em frutos de cupuaçu Theobroma grandiflorum (Willd. ex Spreng) Shum. (Sterculiacea). In: Resumos Congresso Brasileiro de Entomologia, 13, Sociedade Brasileira de Entomologia, Recife, p.137.

Venturieri, G.A. 1993, Cupuaçu: a espécie, sua cultura, usos e processamento. Clube do Cupu, Belém, Pará. 108 p. 